\title{
Differentiation of silane adsorption onto model E-glass surfaces from mixed solutions of amino and glycidyl silanes
}

\author{
X.M.LIU, J.L.THOMASON ${ }^{\dagger}$ and F.R.JONES ${ }^{*}$ \\ Ceramics and Composites Laboratory, University of Sheffield, Sheffield, S1 3JD, UK \\ ${ }^{\dagger}$ European Owens-Corning, Rue de Charneux, Battice, B-4651, Belgium
}

\begin{abstract}
Mixed silanes are often used as coupling agents in sizings for glass fibres. A technique has been developed which enables the preferential adsorption of a particular silane onto a model E-glass from a mixed aqueous silane solution to be probed. It is shown that $\gamma$-Aminopropyltriethoxysilane (APS) is preferentially adsorbed over $\gamma$-glycidoxypropyltrimethoxysilane (GPS) onto model E-glass fibres. High resolution X-ray photoelectron spectroscopy (XPS) has been employed to investigate the nature of silane deposits. Differentiation between the hydrolysed silane deposit and the model silica-based glass substrate was achieved by $\mathrm{Si}_{2 p}$ curve fitting. The extent of total silane adsorption onto model E-glass fibres was determined from the intensity of $\mathrm{CSiO}_{3}$ peak. In the case of APS/GPS mixed silanes, the $\mathrm{N}_{1 \mathrm{~s}}$ peak intensity provides the concentration of APS in the deposit. By comparing the relative intensities of the components in the $\mathrm{Si}_{2 \mathrm{p} 3 / 2}$ and $\mathrm{Si}_{2 \mathrm{p} 1 / 2}$ peaks for $\mathrm{SiO}_{4}$ and $\mathrm{CSiO}_{3}$ with the intensity of the $\mathrm{N}_{1 \mathrm{~s}}$ peak an assessment of differential adsorption proved possible.
\end{abstract}

Key words: E-glass fibres; model surfaces; silanes; coupling agents; X-ray photoelectron spectroscopy (XPS). 


\section{INTRODUCTION}

Organosilanes used as adhesion promoters or coupling agents have a polymer-compatible organic group and three hydrolysable alkoxy functional groups [1, 2]. $\gamma$-Aminopropyltriethoxysilane (APS) and $\gamma$-glycidoxypropyltrimethoxysilane (GPS) are typical examples which provide a substrate, such as a glass fibre surface with compatibility and potential coupling to a polymer resin. They can displace adsorbed water on the glass fibre surface; create a hydrophobic surface of the correct thermodynamic characteristics for complete wetting by the resin and develop strong interfacial bonds between the fibre and resin [3].

Since the 1960s [4], Fourier transform infrared (FTIR), nuclear magnetic resonance (NMR), Raman spectroscopy, X-ray photoelectron spectroscopy (XPS) and secondary ion mass spectrometry (SIMS) have been extensively used to characterize the molecular structures and reactions of silane coupling agents in solution and at composite interfaces. APS has been chosen because of its wide ranging applications as well as the presence of a nitrogen atom which enables a detailed surface analysis [5-13]. Using ToF SIMS Wang et al [10-12] showed that the structure of hydrolysed APS on E-glass consisted of components of differing hydrolytic stability. They also demonstrated that a gradation in the degree of polymerisation accounted for the physisorbed, loosely and strongly chemisorbed layers. The latter was considered to be highly crosslinked and was shown to be rich in aluminum, which could only have originated from the glass surface. GPS has received less attention because of the lack of a diagnostic element. In order to determine the extent of the interaction of GPS with glass surfaces, it is necessary to differentiate between the silicon from the silane deposit and that from the glass substrate. Wagner et al [14] have reported that the difference between the binding energies of the $\mathrm{O} 1 \mathrm{~s}$ and $\mathrm{Si} 2 \mathrm{p}$ lines, $\Delta\left(\mathrm{BE}_{\mathrm{O} 1 \mathrm{~s}}-\mathrm{BE}_{\mathrm{Si2}}\right)$, in inorganic silicon compounds is almost invariant, 429.0 to $429.6 \mathrm{eV}$. Analogously, the binding energy difference, $\Delta\left(\mathrm{BE}_{\mathrm{O} 1 \mathrm{~s}}-\mathrm{BE}_{\mathrm{Si} 2 \mathrm{p}}\right)$, for silicon containing polymers is equally invariant at 429.8 to $430.1 \mathrm{eV}$. Therefore, the use of the difference between the 
binding energies of the $\mathrm{O} 1 \mathrm{~s}$ and $\mathrm{Si} 2 \mathrm{p}$ lines is not a realistic technique for differentiation.

In this work, APS and GPS were adsorbed onto model E-glass fibres from aqueous solutions with a range of composition and concentration. High resolution XPS was employed to characterize APS, GPS and mixed APS/GPS deposits on model E-glass fibres. By comparing the N1s peak intensity with the relative intensities of the components in the $\mathrm{Si} 2 \mathrm{p}_{3 / 2}$ and $\mathrm{Si} 2 \mathrm{p}_{1 / 2}$ peaks at differing binding energies for $\mathrm{SiO}_{4}$ and $\mathrm{CSiO}_{3}$, we have determined the adsorption isotherm for APS and GPS on model E-glass surfaces.

\section{EXPERIMENTAL}

2.1 Materials and Sample Preparation

E-glass fibres used in this work were unsized E-glass fibres supplied from Owens-Corning. Model E-glass fibres were prepared by washing E-glass fibres with nitric acid (69\%, analytical reagent, Aldrich) for 6 days at room temperature to extract non-silicon elements, which otherwise interfere with the XPS analysis, and provide a silica/silanol rich surface, which represents the outermost surface of the fibre.

Fresh silane coupling agents, APS (A-1100) and GPS (A-187) were provided by GE Speciality Materials and used as received without further purification. The chemical structures of APS and GPS are given in Scheme 1.

$\mathrm{NH}_{2}-\left(\mathrm{CH}_{2}\right)_{3}-\mathrm{Si}-\left(\mathrm{OC}_{2} \mathrm{H}_{5}\right)_{3}$

I APS

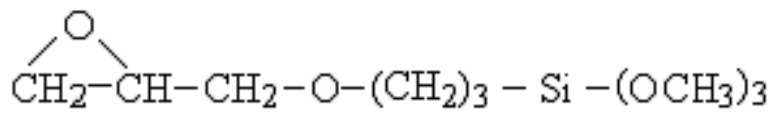

II GPS

Scheme 1. Chemical structure of APS (I) and GPS (II). 
Aqueous silane solutions with the following concentrations $0.1 \%, 0.5 \%$ and $1.0 \%$ by weight respectively were used to silanize the surface of these model E-glass fibres. APS was hydrolysed in deionised water. GPS was hydrolysed at $\mathrm{pH} 4.0$ in the presence of aqueous acetic acid (Aldrich, UK). Mixed APS/GPS (1:1) solutions were prepared by dissolving APS in deionised water and adjusting the $\mathrm{pH}$ to 4 with acetic acid. Subsequently GPS was added and the $\mathrm{pH}$ adjusted, if necessary, by the addition of a further aliquot of acetic acid.

The model E-glass fibres (after nitric acid treatment) were immersed in the solutions of APS, GPS and APS/GPS (1:1) for 15 minutes at room temperature. Then they were washed with fresh deionised water three times and dried in a vacuum oven at $50^{\circ} \mathrm{C}$ for 24 hours [15].

\subsection{XPS analysis}

High resolution XPS was carried out using the SCIENTA ESCA 300 spectrometer at the National Centre for Surface Spectroscopy (NCESS), Daresbury, UK. Photoelectrons were excited by the monochromatic Al K $\alpha$ X-ray source ( $h v=1486.7$ $\mathrm{eV}$ ). The electron analyzer was used in the high transmission mode, with the pass energy of $75 \mathrm{eV}$ for both wide scans and narrow scans of selected regions. The electron analyzer energy slit width was set at $1.9 \mathrm{~mm}$ during wide scans and at 0.8 $\mathrm{mm}$ for the narrow scans. Charge compensation was achieved using an electron flood gun of 4-6 eV. The instrument operating pressure during analysis was less then $10^{-8}$ mbar. For each sample the XPS spectra were recorded at $45^{\circ}$ take-off-angle.

$\mathrm{O} 1 \mathrm{~s}(533.0 \mathrm{eV})$ was employed as a reference for the calculation of the binding energy. The relative concentrations of all the elements, present in the wide scans, were calculated using the atomic sensitivity factors specific to the SCIENTA instrument. The curve-fitting procedure was carried out, after background subtraction, assuming a complex mixed Gaussian-Lorentzian peak shape, with a tail function that was dependent on the photoelectron energy [15]. 


\section{RESULTS AND DISCUSSION}

\subsection{Model E-glass fibres}

Figure 1 shows the survey spectra of the untreated E-glass fibres and model E-glass fibres after nitric acid treatment. The relative surface compositions of these two surfaces are given in Table 1. As seen from the survey spectra (Figure 1) and the atomic concentrations (Table 1), the carbon signal is low and results from small degree of a contamination. The nitric acid treatment removed the metal ions components such as $\mathrm{Al}$ and $\mathrm{Ca}$ from the E-glass surfaces (at least to a depth corresponding to the take-off-angle of $45^{\circ}$ ) and produced a model silica-rich surface composed of mainly Si-O and Si-OH.

\section{Table 1.}

Atomic concentrations (at \%) of untreated and acid-treated E-glass fibres determined from the survey scan at a take-off-angle of $45^{\circ}$.

\begin{tabular}{lccccc}
\hline & $\mathrm{O}$ & $\mathrm{C}$ & $\mathrm{Si}$ & $\mathrm{Ca}$ & $\mathrm{Al}$ \\
\hline Untreated E-glass fibres (at \%) & 57.0 & 16.9 & 15.8 & 4.5 & 5.8 \\
Model E-glass fibres (at \%) & 61.6 & 10.8 & 27.6 & 0.0 & 0.0 \\
\hline
\end{tabular}

\subsection{APS coated model E-glass fibres}

Figure 2 shows the survey spectrum and the high resolution spectra of $1 \%$ APS coated model E-glass fibres. The N1s signal is observed from the APS deposit at a binding energy of approx. $400 \mathrm{eV}$. The ratio of $\mathrm{N} / \mathrm{Si}$ is around 0.1 , which is lower than 1 for APS, indicating that the thickness of the APS coating formed on model E-glass fibres is thinner than the analysis depth of XPS. Thus, all the peaks in the spectra can be attributed to both the APS deposit and the E-glass substrate. 

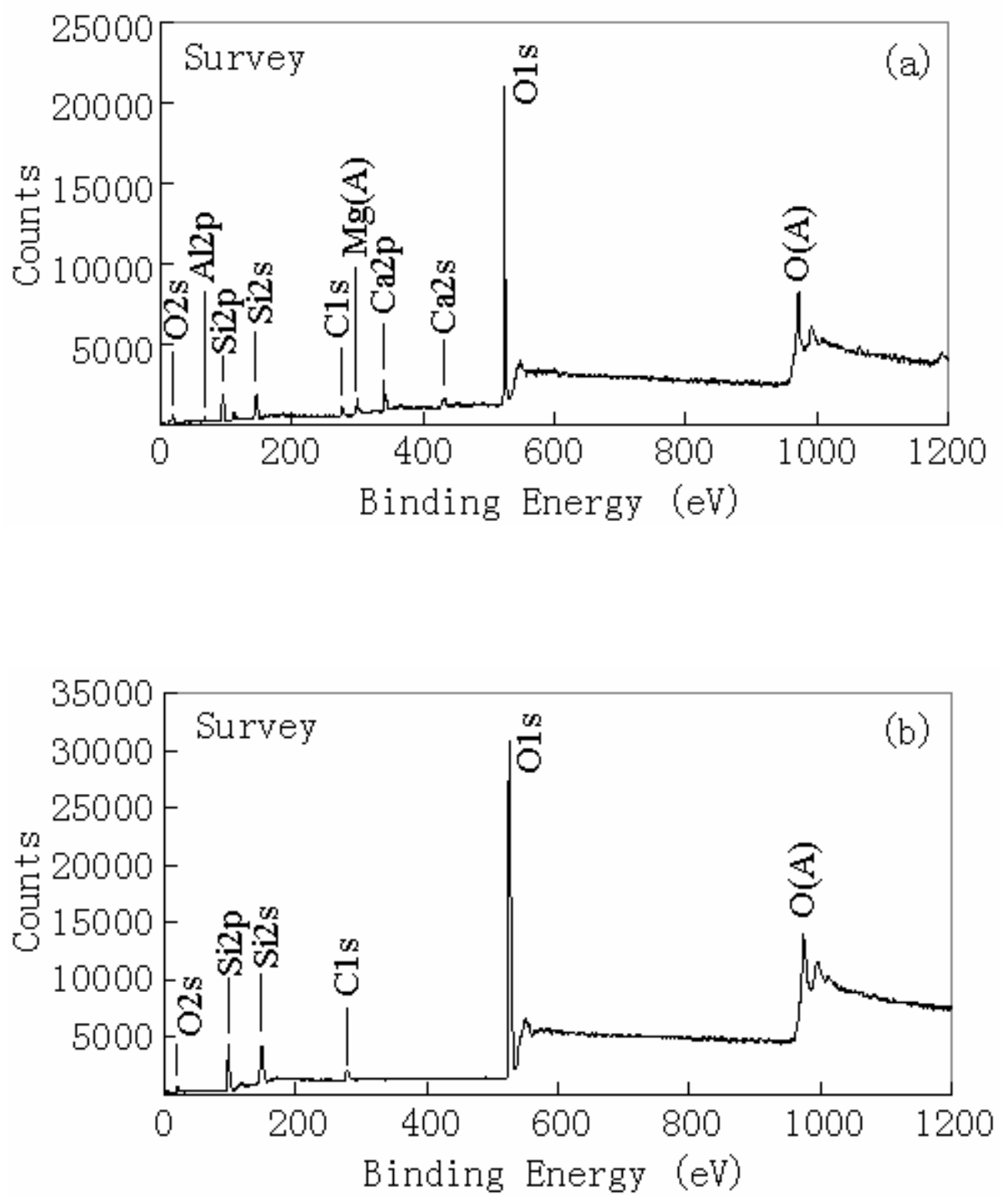

Figure 1. XPS wide scan spectra of (a) untreated E-glass fibres and (b) model E-glass fibres. An A in parenthesis denotes Auger line

As seen in Figure 2 (b), the N1s spectrum can be fitted with two components, which are assigned to amino groups in (i) free $-\mathrm{NH}_{2}(399.6 \mathrm{eV}, 61 \%)$ and (ii) protonated $-\mathrm{NH}_{3}{ }^{+}(401.4 \mathrm{eV}, 39 \%)$. These binding energy assignments are in a good agreement with those reported by Horner et al [16], in a high resolution study of hydrolysed APS deposited on a range of metal substrates, and with other literature reports [17-19]. A possible explanation for the formation of protonated amino groups on the glass 
surface is that the amine is protonated by the hydroxyl groups $(\mathrm{Si}-\mathrm{OH})$ present in the siloxane layer as well as on the glass surface. The presence of significant amounts of hydroxyls on the unsilanised glass surface has been confirmed by the ToF SIMS studies of Wang et al [12].
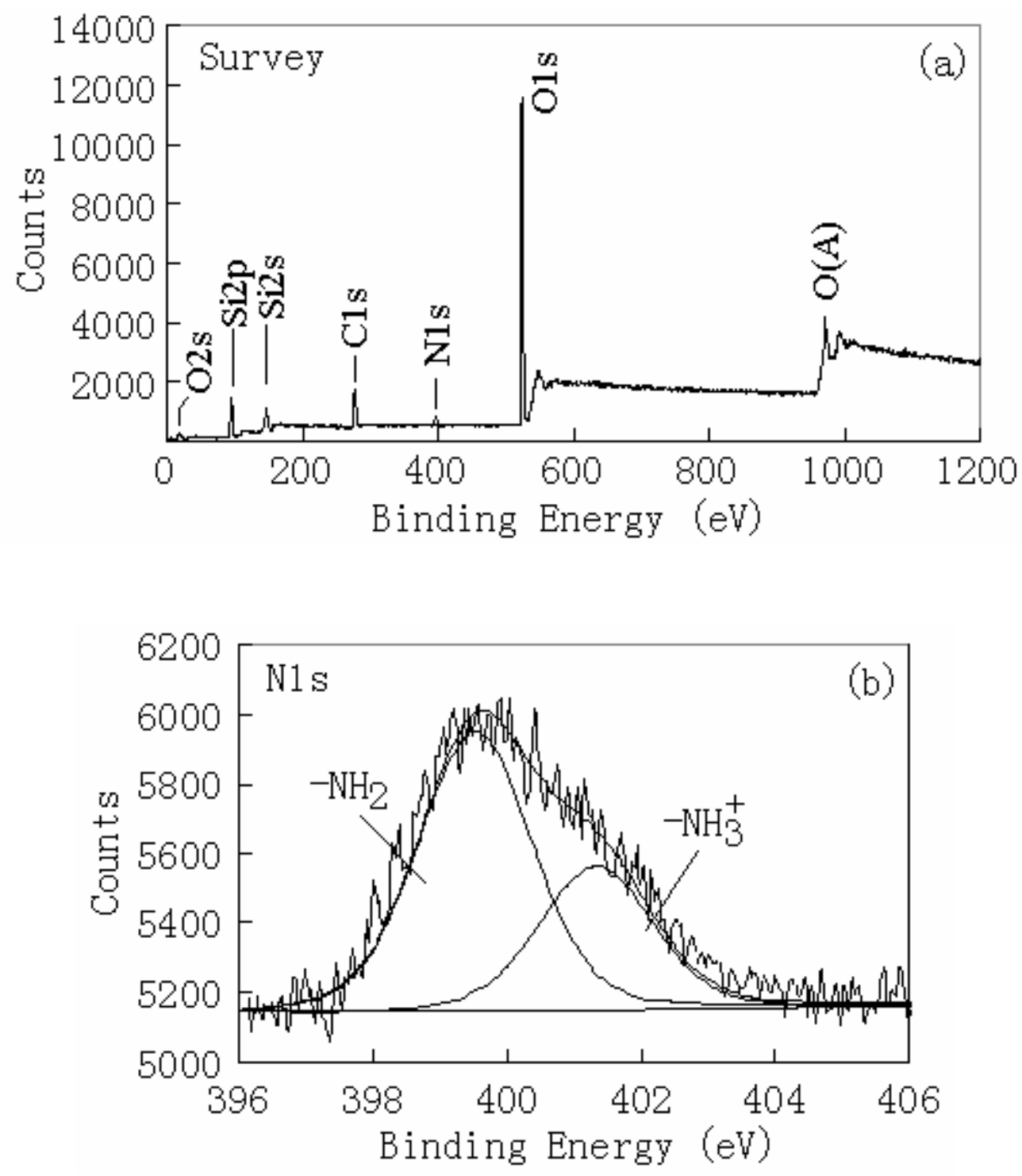

Figure 2. XPS analysis of the 1\% APS coated model E-glass fibres: (a) survey and (b) narrow scan of the $\mathrm{N} 1 \mathrm{~s}$ region. An A in parenthesis denotes Auger line

The Si $2 p$ spectra for the model E-glass fibres and 1\% APS coated model E-glass fibres are shown in Figure 3. As seen in Figure 3a, the Si2p spectrum for model 
E-glass fibres is fitted with two peaks at 103.5 and $104.2 \mathrm{eV}$, which represent the $2 \mathrm{p}$ doublets associated with $\mathrm{SiO}_{4}$. After APS treatment, the shape of Si $2 \mathrm{p}$ peak changed. Therefore, the Si $2 p$ peak (Figure $3 b$ ) had to be fitted with four components. The peaks at 102.4 and $103.1 \mathrm{eV}$ are attributed to a siloxane bond $\mathrm{CSiO}_{3}$. The percentage of siloxane component $\left(\mathrm{CSiO}_{3}\right)$ is about $12 \%$. Therefore, in $\mathrm{Si} 2 \mathrm{p}$ spectra, it is possible to separate the siloxane $\left(\mathrm{CSiO}_{3}\right)$ caused by the silane deposit from $\mathrm{SiO} 4$ of the model glass substrate.
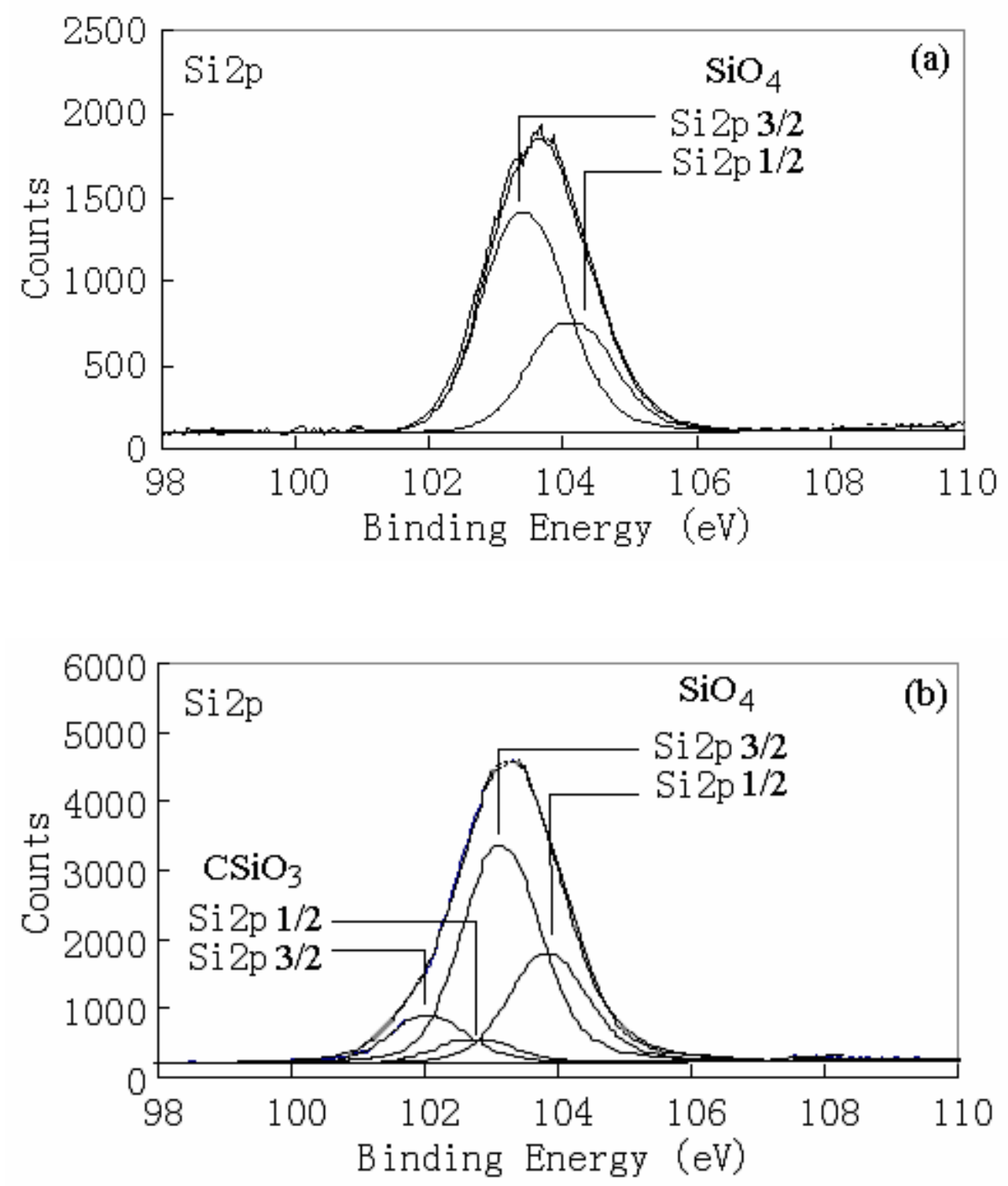

Figure 3. The Si2p curve fittings for (a) model E-glass fibres and (b) $1 \%$ APS coated model E-glass fibres. 
Using the semi-quantitative XPS data, the atomic concentrations of APS in the hydrolysed deposit is given in Figure 4 as a function of initial APS concentration. With an increase in the concentration of APS in the coating solution, the N1s intensity increased, indicating that the thickness of the deposit on the model E-glass fibres was an approximately linear function of the concentration of APS solution. The Si2p intensity decreases because the coating has a lower concentration than the substrate, which confirms that the thickness of the deposit increased. The intensity of $\mathrm{CSiO}_{3}$ is also indicative of the contribution of the APS deposit to spectrum intensity. Thus the number of monolayers of APS which are adsorbed from the solution increases with a higher silane concentration. There is a good correlation between the APS isotherm determined from the Si2p and N1s analysis. According to the chemical structure of APS (I), its hydrolysed form has $\mathrm{CSiO}_{3}$ and $\mathrm{NH}_{2}$ groups in 1:1 ratio. Thus, it is reasonable to observe similar intensities for $\mathrm{CSiO}_{3}$ and $\mathrm{N} 1 \mathrm{~s}$. We conclude therefore that it is possible to differentiate the silicon signals arising from the model E-glass fibres and that from the APS silane deposit.

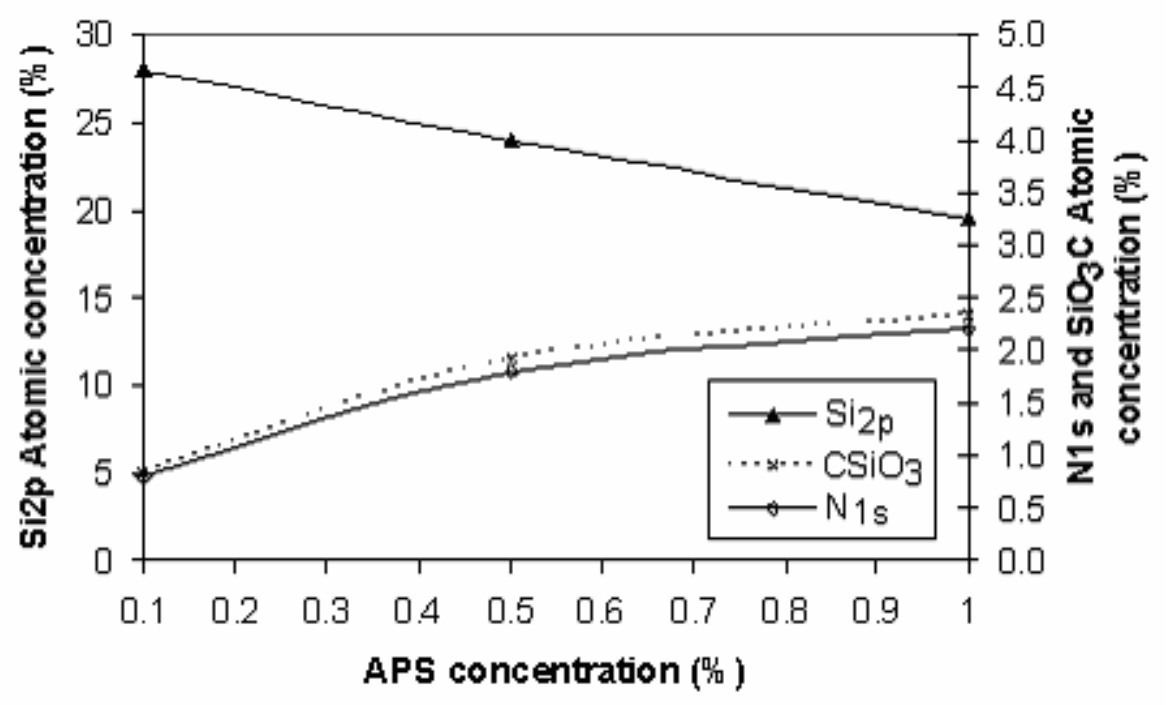

Figure 4. Atomic concentrations of $\mathrm{Si} 2 \mathrm{p}, \mathrm{N} 1 \mathrm{~s}$ and $\mathrm{CSiO} 3$ versus the initial concentration of APS in solution. 


\subsection{GPS coated model E-glass fibres}

Figure 5 shows XPS spectra obtained from 1\% GPS coated model E-glass fibres. Figure $5 \mathrm{~b}$ gives the curve fitting of Si2p signal, which contains Si doublet peaks for $\mathrm{CSiO}_{3}$ from silane deposit at lower binding energies (102.4 eV and $\left.103.1 \mathrm{eV}\right)$ and $\mathrm{Si}$ doublet peaks for $\mathrm{SiO}_{4}$ from the model E-glass substrate at higher binding energies (103.5 eV and $104.2 \mathrm{eV})$.
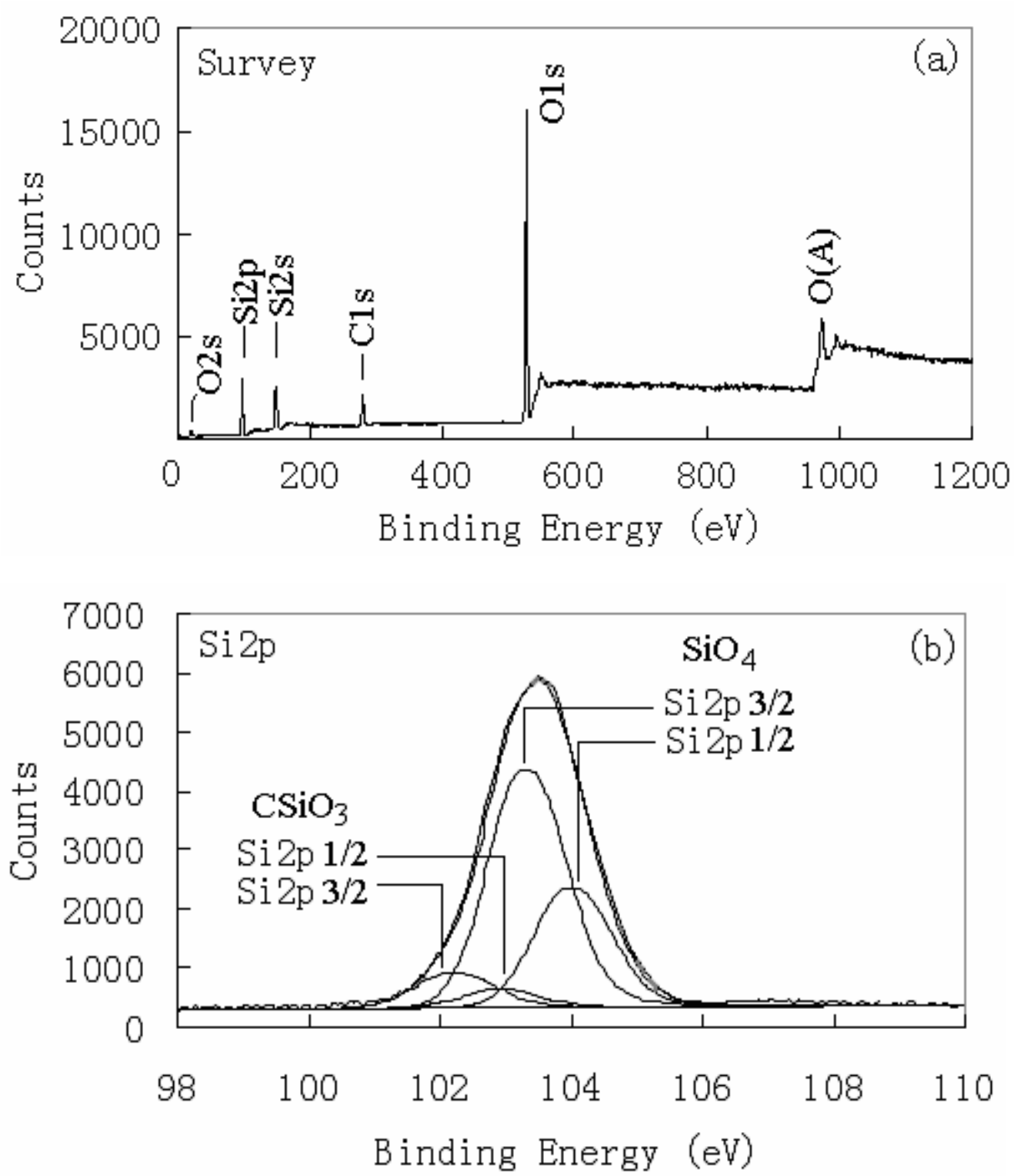

Figure 5. XPS analysis of the 1\% GPS coated model E-glass fibres: (a) survey and (b) narrow scan of the Si2p region. An A in parenthesis denotes Auger line 
Table 2 gives the quantitative XPS results on the different concentration GPS coated model E-glass fibres. The atomic concentrations obtained from the wide scans (Table 2) are in good agreement with those calculated from the percentage of Si $2 p$ from GPS deposit $\left(\mathrm{CSiO}_{3}\right)$ and the model E-glass substrate $\left(\mathrm{SiO}_{4}\right)$ (Table 3). For example, for every $100 \mathrm{Si}$ atoms detected from the 1\% GPS coated model E-glass fibres, 89 are from $\mathrm{SiO}_{4}$ and 11 are from $\mathrm{CSiO}_{3}$. We should therefore also detect $178 \mathrm{O}$ atoms from the surface, $38.5 \mathrm{O}$ atoms from the silane, and $66 \mathrm{C}$ atoms from the silane. Thus we can calculate the atomic percentages of $\mathrm{O}, \mathrm{C}$ and $\mathrm{Si}$ to be $56.6 \%, 17.3 \%$, and $26.1 \%$. These calculated values are in excellent agreement with the experimental values obtained from the survey spectra. The calculated $1 \mathrm{~s}$ intensity of $0.1 \%$ GPS coated model E-glass fibres is slightly lower than that obtained from the wide scan spectrum since only a molecularly thin coating is deposited from a $0.1 \%$ GPS. There is a high probability that it could be inhomogeneous. Furthermore the carbon contamination on the model E-glass fibres may also contribute to the XPS spectrum. It is not clear if the patchiness is associated with the carbon contamination and this needs further research.

Figure 6 plots the atomic concentrations of GPS on the coated model E-glass fibres calculated from XPS survey spectrum against the atomic concentrations obtained from $\mathrm{Si} 2 \mathrm{p}$ curve fitting as a $\mathrm{SiO} 4: \mathrm{CSiO} 3$ ratio. All the data points follow the line which represents a $1: 1$ ratio of $\mathrm{SiO} 4: \mathrm{CSiO} 3$. It indicates that the atomic concentrations obtained from survey spectra are in excellent agreement with the values calculated from the curve fitting of Si2p peak. This Si2p curve fitting has been successfully used to determine the adsorption isotherm for GPS onto an E-glass surface. 
Table 2.

Atomic concentrations (at \%) of GPS coated model E-glass fibres determined from the survey scan at a take-off-angle of $45^{\circ}$ and the fraction (\%) of Si2p signal associated with GPS $\left(\mathrm{CSiO}_{3}\right)$ and the model E-glass fibres $\left(\mathrm{SiO}_{4}\right)$.

\begin{tabular}{cccc||cc}
\hline GPS Concentration (\%) & $\mathrm{O}$ & $\mathrm{C}$ & $\mathrm{Si}$ & $\mathrm{SiO}_{4}$ & $\mathrm{CSiO}_{3}$ \\
\hline 0.1 & 62.2 & 10.5 & 27.3 & 98 & 2 \\
0.5 & 57.1 & 16.1 & 26.8 & 93 & 7 \\
1.0 & 55.7 & 19.1 & 25.2 & 89 & 11 \\
\hline
\end{tabular}

\section{Table 3.}

Atomic concentrations (at $\%$ ) of GPS coated model E-glass fibres calculated from the percentage of $\mathrm{Si} 2 \mathrm{p}$ from GPS deposit $\left(\mathrm{CSiO}_{3}\right)$ and the model E-glass substrate $\left(\mathrm{SiO}_{4}\right)$.

\begin{tabular}{cccc}
\hline GPS Concentration $(\%)$ & $\mathrm{O}$ & $\mathrm{C}$ & $\mathrm{Si}$ \\
\hline 0.1 & 64.4 & 3.8 & 31.7 \\
0.5 & 59.7 & 11.9 & 28.4 \\
1.0 & 56.6 & 17.3 & 26.1 \\
\hline
\end{tabular}




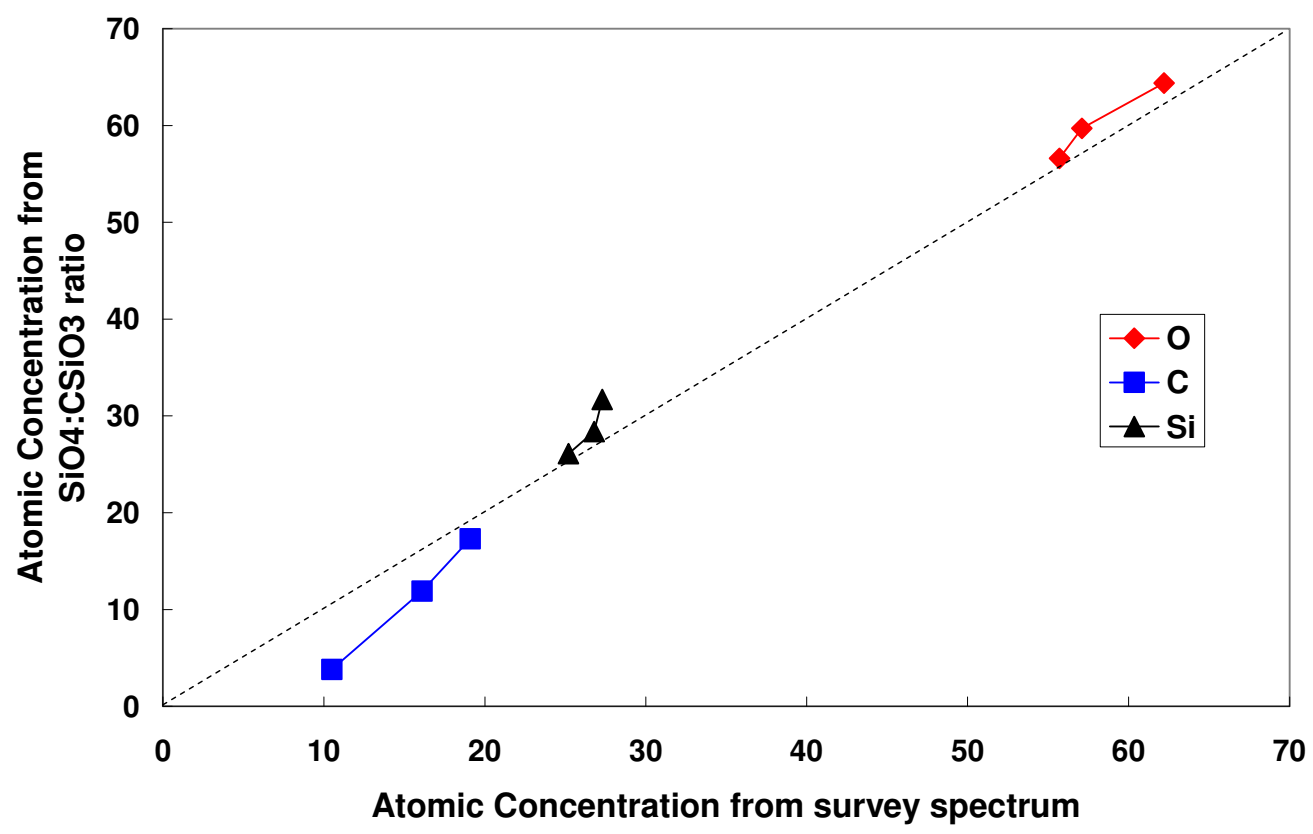

Figure 6. Plot of atomic concentration obtained from $\mathrm{SiO}_{4}: \mathrm{CSiO}_{3}$ ratio vs. atomic concentration (at $\%$ ) from the XPS survey spectrum for the concentrations of the GPS solutions employed for coating (see Table 3).

\subsection{APS/GPS mixed silane coated model E-glass fibres}

The wide scan and high resolution Si $2 \mathrm{p}$ spectra for a $1.0 \%$ APS/GPS mixed silane on the glass surface are given in Figure 7. The detection of an N1s signal shows that APS is involved in the mixed silane deposit. Si2p peak has been fitted confidently with the components from silane coating $\left(\mathrm{CSiO}_{3}\right)$ which arises from both APS and GPS and from model E-glass substrate $\left(\mathrm{SiO}_{4}\right)$. The $\mathrm{CSiO}_{3}$ component fraction is about $15 \%$, which is slightly higher than that obtained from $1.0 \%$ APS (12\%) and 1\% GPS (11\%) deposits alone. It appears that the adsorption of silanes from aqueous solution onto model E-glass fibres is promoted when mixed silanes are used. 

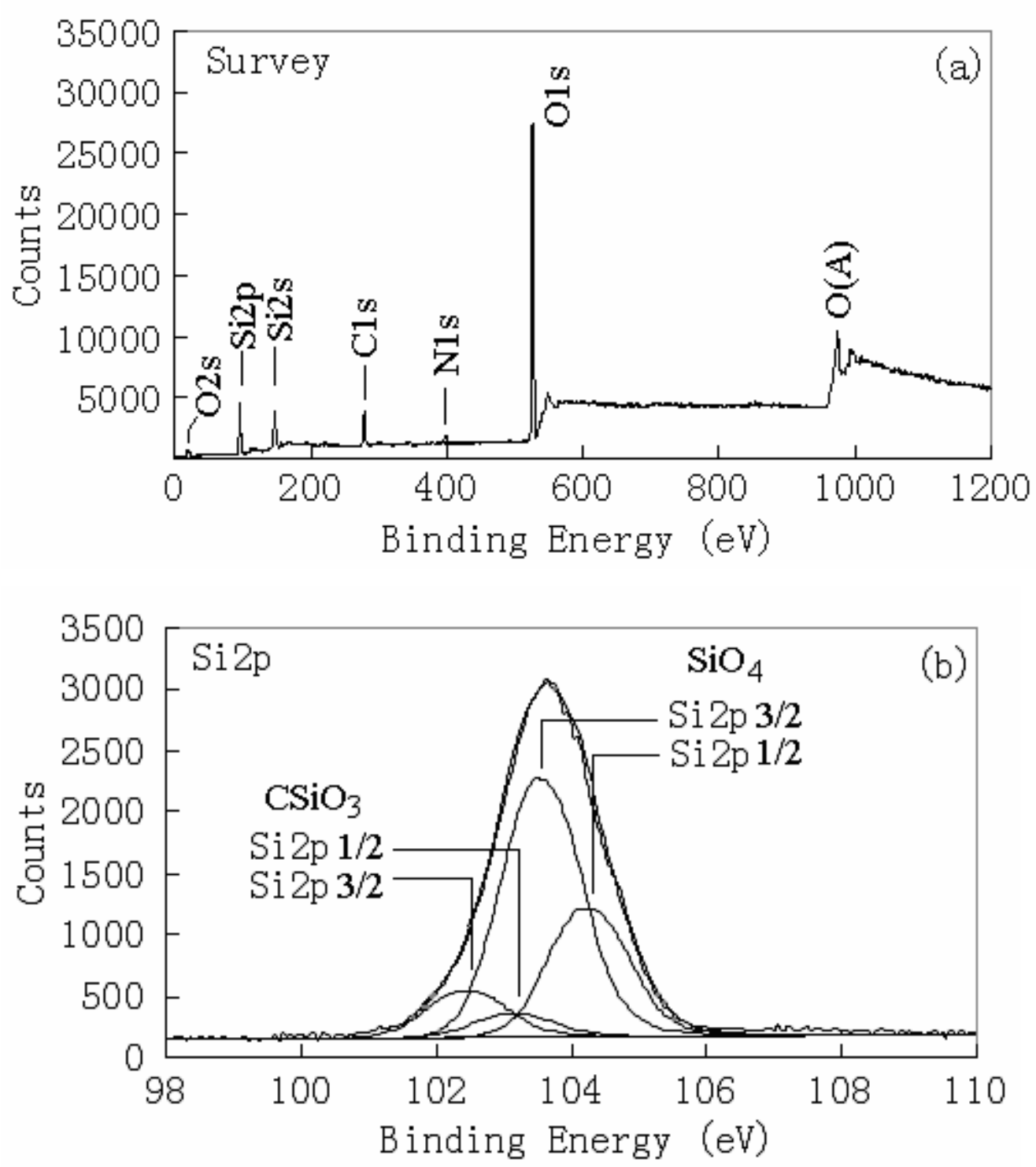

Figure 7. XPS analysis of the 1\% APS/GPS mixed silane coated model E-glass fibres: (a) survey and (b) narrow scan of the Si2p region. An A in parenthesis denotes Auger line

The adsorption isotherms for APS and GPS on model E-glass fibres which are shown in Figure 8 have been obtained from the XPS data for deposition from different concentrations of mixed APS/GPS silane. APS is the only source of the N1s signal and there is $1 \mathrm{~mol}$ of $-\mathrm{NH}_{2}$ and $1 \mathrm{~mol}$ of $\mathrm{CSiO}_{3}$ in $1 \mathrm{~mol}$ of APS. Therefore the atomic concentration of N1s signifies the percentage of adsorbed APS in the mixed silane coating. The atomic concentration of $\mathrm{CSiO}_{3}$ represents the proportion of $\mathrm{Si} 2 \mathrm{p}$ originating from both APS and GPS in the deposit. Thus the difference between the 
atomic concentrations of $\mathrm{CSiO}_{3}$ and N1s provides the percentage of GPS in the mixed silane coating. The $\mathrm{N} 1 \mathrm{~s}$ and $\mathrm{CSiO}_{3}$ concentration and the adsorption ratio of APS to GPS from the solutions of mixed silanes in a range of composition are shown in Table 4.

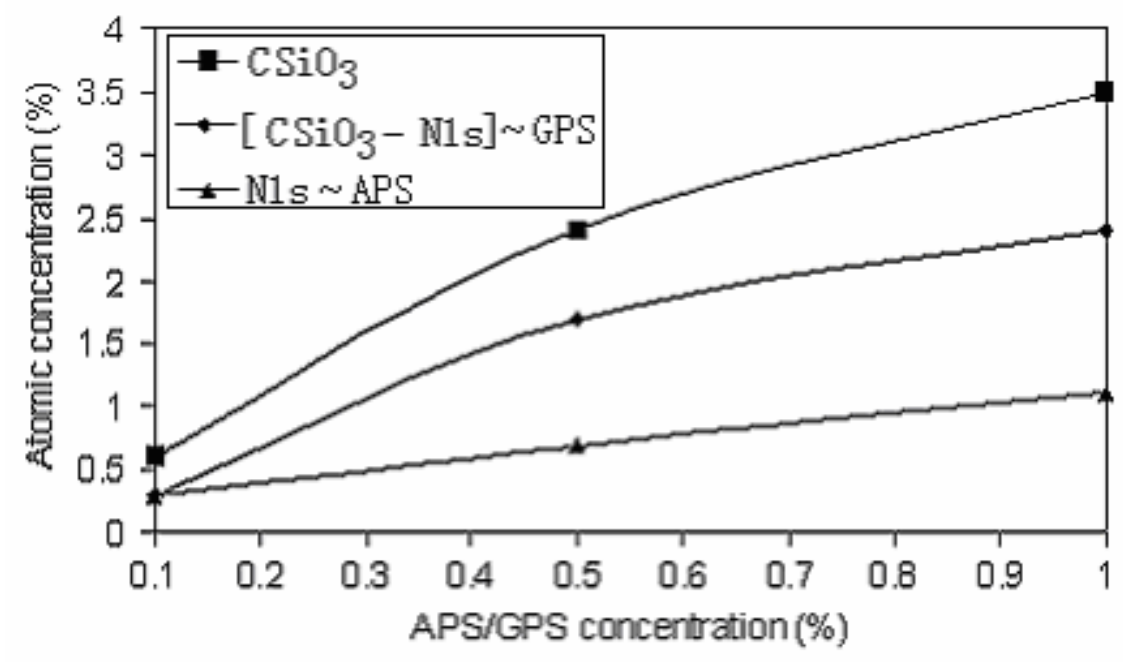

Figure 8. The adsorption isotherms for APS and GPS on model E-glass fibres obtained by plotting atomic concentrations of $\mathrm{CSiO} 3$ from the mixed silane deposits, N1s from APS and the difference between them (CSiO3-N1s) from GPS versus the initial concentration of the mixed 1:1 APS/GPS solution.

\section{Table 4.}

$\mathrm{N} 1 \mathrm{~s}$ and $\mathrm{CSiO}_{3}$ concentrations and APS to GPS adsorption ratios from mixed silane coated model E-glass fibres determined from XPS spectra at a take-off-angle of $45^{\circ}$.

\begin{tabular}{cccc} 
APS/GPS Concentration (\%) & $\mathrm{N} \mathrm{1s}(\%)$ & $\mathrm{CSiO}_{3}(\%)$ & GPS/APS ratio \\
\hline 0.1 & 0.3 & 0.6 & 1.0 \\
0.5 & 0.7 & 2.4 & 2.4
\end{tabular}


As seen in Figure 8 and Table 4, APS and GPS are adsorbed onto model E-glass surfaces at 1:1 ratio from $0.1 \%$ APS/GPS mixed silane solution, which is similar with the initial ratio of APS to GPS in the solution. When the mixed silane concentration was increased to $0.5 \%$ and $1.0 \%$, GPS and APS were detected in the XPS spectra at a ratio $\approx 2: 1$. One possible reason is that the siloxane layer is homogeneous with higher GPS concentration than APS. Another possibility is that APS is adsorbed preferentially over GPS onto the model E-glass fibre, so that as the concentration of GPS in the mixed solution increases, it will be deposited on top of the initial layer. Alternatively GPS can react with the APS through its well known epoxy amine chemistry [20]. Experiments are planned to investigate the orientation of APS and GPS by using angle resolved XPS. In section 3.2, we demonstrated that (at 1\%) the silane layer was thinner than the analysis depth for XPS, indicating that we should easily detect all of the APS layer under the GPS without any loss in signal. Therefore, the differences detected must arise from the real differences in adsorption potential of APS and GPS onto E-glass like surfaces.

\section{CONCLUSIONS}

In this paper, the interaction of amino and glycidyl silanes with nitric acid treated E-glass fibres has been studied by high resolution XPS. When APS and GPS are deposited onto a model E-glass surface, the Si2 $\mathrm{p}_{3 / 2}$ and $\mathrm{Si} 2 \mathrm{p}_{1 / 2}$ peaks can be fitted with the components from the silane at the binding energies of $102.4 \mathrm{eV}$ and $103.1 \mathrm{eV}$ and with the components from the substrate at the binding energies of $103.5 \mathrm{eV}$ and $104.2 \mathrm{eV}$. Therefore, it is possible to differentiate between the Si contribution from the silane $\left(\mathrm{CSiO}_{3}\right)$ and from the substrate $\left(\mathrm{SiO}_{4}\right)$, and determine the extent of GPS adsorption onto model E-glass surfaces. The formation of protonated amine groups in the APS deposit has also been detected by N s curve fitting. 
For the mixed APS/GPS silane solutions, the adsorption isotherms for APS and GPS on the model E-glass surfaces have been obtained by comparing the N1s peak intensity with the relative intensities of the components in the $\mathrm{Si} 2 \mathrm{p}$ peaks for $\mathrm{SiO}_{4}$ and $\mathrm{CSiO}_{3}$. APS and GPS are equally adsorbed onto model E-glass surfaces from $0.1 \%$ APS/GPS mixed silane solution. When the concentration of mixed silane increased to $0.5 \%$ and $1.0 \%$, more GPS than APS is detected in the siloxane layer.

\section{ACKNOWLEDGEMENTS}

The authors would like to thank Owens-Corning for financial support and for supply of glass fibres. The authors acknowledge EPSRC funding for access to the NCESS facility at Daresbury Laboratory. The authors are members of the Ceramics and Composites Laboratory which is EPSRC funded.

\section{REFERENCES}

[1] Plueddemann E P, Silane coupling agents, $2^{\text {nd }}$ Ed., New York, Plenum Press, 1991.

[2] Mittal K L, Silanes and other coupling agents, Utrecht: VSP, 1992.

[3] Jones F R, “Glass Fibres", High-performance Fibres, Hearle J W S (ed.), New York, pp. 191-235.

[4] Schrader M E, Lerner I and D’Oria F J, Modern Plastics, vol. 45 (1967) 195.

[5] Ishida H and Koenig J L, J. Coll. Interface Sci., 64 (1978) 565.

[6] Ishida H and Koenig J L, J. Polym. Sci., Polym. Phys. Ed., 17 (1979) 1807.

[7] Ishida H and Koenig J L, J. Polym. Sci., Polym. Phys. Ed., 18 (1980) 1931.

[8] Chiang C H, Ishida H and Koenig J L, J. Coll. Interface Sci., 74 (1980) 396.

[9] Ishida H, Naviroj S, Tripathy S, Fitzgerald J J and Koenig J L, J. Polym. Sci., Polym. Phys. Ed., 20 (1992) 701.

[10] Wang D, Jones F R and Denison P, J. Adhesion Sci. and Tech., 6 (1992) 79.

[11] Wang D, Jones F R and Denison P, Surface Interface Analysis, 20 (1993) 457. 
[12] Wang D and Jones F R, J. Mater. Sci., 28 (1993) 2481.

[13] Jones F R, Interfacial Phenomena in Composite Materials 1989, Jones F R (ed.), London, Butterworths, 1989, pp. 25-32.

[14] Wagner C D, Passoja D E, Hillery H F, Kinisky T G, Six H A, Jansen W T and Taylor J A, J. Vac. Sci. Technol., 21 (1982) 993.

[15] T. Choudhury and F.R. Jones, International Journal of Adhesion and Adhesives, $26(2006) 79$.

[16] Horner M R, Boerio F J and Clearfirld H W, Silane and other coupling agents, Mittal K L (ed.), VSP, 1992, pp. 241.

[17] Eldridge B N, Buchwalter L P, Chess C A, Goldberg M J, Goldblatt R D and Novak F P, Silane and other coupling agents, Mittal K L (ed.), VSP, 1992, pp. 304.

[18] Wesson S P, Jen J S and Nishioka G M, Silane and other coupling agents, Mittal K L (ed.), VSP, 1992, pp. 379.

[19] Beamson G and Briggs D, "High resolution XPS of Organic Polymers", The Scienta ESCA300 Database, John Wiley \& Sons, 1992.

[20] B.Ellis (Ed.), Chemistry and Technology of Epoxy Resins. Chapman \& Hall, Glasgow (1993). 